\title{
A Region-Aware Multi-Objective Auto-Tuner for Parallel Programs
}

\author{
Klaus Kofler, Juan J. Durillo, Philipp Gschwandtner, and Thomas Fahringer \\ University of Innsbruck, Institute of Computer Science \\ Distributed and Parallel Systems Group \\ Technikerstrasse 21a, 6020 Innsbruck, Austria \\ \{klaus, juan, philipp, tf $\} @$ dps.uibk.ac.at
}

\begin{abstract}
Auto-tuning has become increasingly popular for optimizing non-functional parameters of parallel programs. The typically large search space requires sophisticated techniques to find well performing parameter values in a reasonable amount of time. Different parts of a program often perform best with different parameter values. We therefore subdivide programs into several regions, and try to optimize the parameter values for each of those regions separately as opposed to setting the parameter values globally for the entire program. As this enlarges the search space even further, we have to extend existing autotuning techniques in order to obtain good results. In this paper we introduce a novel enhancement to the RS-GDE3 algorithm which is used to explore the search space for auto-tuning programs with multiple regions regarding several objectives. We have implemented our auto-tuner using the Insieme compiler and runtime system. In comparison to a non-optimized parallel version of the tested programs, our novel approach achieves up to 7.6, 10.5, and 61.6 fold improvements for three tuned objectives wall time, energy consumption, and resource usage, respectively.
\end{abstract}

\section{INTRODUCTION}

Optimizing parallel programs becomes increasingly difficult with the rising complexity of modern parallel architectures which includes, for example, a dramatic increase of the number of cores per chip or the availability of multi-level partially shared cache hierarchies. Automatic software tuning, or simply auto-tuning [1], arose as an attempt to better exploit hardware features by automatically tuning applications. An auto-tuner tries to find promising configurations for a given program. A configuration consists of a set of non-functional parameters with a corresponding value range which can influence a program's performance by transforming the source code of the application, or by finding the right parameter values that govern the execution of an application on a given architecture (e.g. number of threads, frequency per core, etc.).

This paper describes a novel auto-tuning approach for programs with multiple single-entry single-exit code regions whose non-functional behavior depends on at least one tunable parameter. We assume that we can measure the non-functional behavior of these regions for the optimization objectives (e.g. wall time, energy consumption, etc.). Tuning multi-region applications exposes additional challenges for auto-tuning techniques. Firstly, there usually does not exist a single set of parameter values which is optimal for all the regions of a program. However, setting the parameter values individually for every region leads to a huge search space as it grows exponentially with the number of tuning opportunities, i.e. the number of regions. Secondly, the execution of a region may be influenced by the parameter values applied to neighboring regions. Previous work [2] observed that the optimal parameter values for individual regions of hybrid MPI/OpenMP applications led to sub-optimal overall performance.

Auto-tuning techniques are widely used [3], [4], [5], [6], [7], [8], [9], [10], [11] but are often limited to using the same parameter values for every region, i.e. globally for the entire program, ignoring the fact that different parts of the code may benefit from specific parameter values.

Our auto-tuner can optimize a generic set of objectives which do not necessarily correlate with each other. This lack of correlation makes it impossible to find a single solution which is best in every objective. For example, if some regions are optimized for one objective while others are optimized for different objectives, it is very likely that the overall performance of the program will suffer. It can happen that a configuration exposes small execution time at the cost of high resource usage for one region, but the contrary for another region, resulting in sub-optimal overall performance.

The approach proposed in this paper extends the method presented in [12], which is limited to optimizing each region in isolation, by adding region-aware auto-tuning support for the entire program. The contributions of this paper are the following:

- A region-aware multi-objective auto-tuner.

- A compiler-runtime system that automatically identifies regions and enables automatic tuning of their parameters.

- Evaluation of several global and region-aware auto-tuning strategies for several codes on different target architectures which demonstrates the importance of region-aware auto-tuning compared against global optimization.

The rest of this paper is organized as follows: Section II exemplary shows the inherit advantages of region-aware autotuning while Section III gives detailed insights on the autotuning approach presented in this paper. The implementation details of the presented work can be found in Section IV while Section V details the metrics that we use to compare the quality of the different auto-tuning approaches. The results of the experiments performed in this paper are shown in Section VI, whereas Section VII discusses some aspects of the obtained results in detail. Section VIII gives an overview 
of the related work and the conclusion of our findings are summarized in Section IX.

\section{Motivation}

In this section, we motivate the need for region-aware autotuners by using a simple example program consisting of two regions. Both regions perform a parallel matrix multiplication. In the first region, only the outermost loop is parallelized; in the second, we parallelize only the innermost loop. As a consequence we have two regions with different execution behavior: the first one scales well with the number of threads and the second one does not. In order to have similar execution times for both regions, the matrix size in the second region is only one quarter of the matrices in the first region.

The experiments in this section are performed on the Ivy Bridge-EP architecture described in Section VI. Our goal is to find the optimal configuration for executing this program. For the sake of performing an exhaustive search of all possible program configurations, we assume that these regions only expose the number of threads as a tunable parameter.

We select configurations for the previously described program using three different approaches:

- Isolated: This approach optimizes both regions in isolation.

- Global: This approach is constrained to find a single set of parameter values to be used in both regions.

- Region-Aware: This approach optimizes both regions using individual parameter values for each of them to maximize the program's overall performance.

The best configurations found by these approaches are summarized in Table I. The first two rows of the table show the number of threads for each region chosen by the corresponding approach — which can be found in the different columns. The following two rows include the execution time for each of these regions with the aforementioned indicated number of threads. The fifth row shows the program's execution time when the regions are configured as indicated in the first two rows. Finally, the last row shows the relative difference regarding the best found execution time across all three approaches.

The worst execution time corresponds to the Isolated approach. The reason is that it will run the first region with a large number of threads, as it does scale well. However, this has a negative effect on the second region, which does not scale well. The change from 20 to only a few threads between the regions introduces a significant overhead by the underlying runtime system, as it also implies a change of the number of sockets where computations are performed on. The fastest option for the second region is to use two threads, taking 5798ms. Using for example 7 threads, which is the fastest option after the first region has been executed with 10 threads, results in an execution time of $6387 \mathrm{~ms}$ in this scenario.

The Global approach yields better results since the code regions are not optimized in isolation but regarding the overall program performance. However, as this approach is limited to the same parameter values for both regions, it is unable to exploit the full potential of the hardware. This drawback is overcome by the Region-Aware approach. Besides taking into account the overhead for a change in using different socket numbers, it can customize the regions to their needs for hardware resources.

TABLE I: Theoretical potential of different auto-tuning approaches, determined by exhaustive search for an example program with two regions.

\begin{tabular}{l|ccc} 
& Isolated & Global & Region-Aware \\
\hline \#Threads Region 1 & 20 & 10 & 10 \\
\#Threads Region 2 & 2 & 10 & 7 \\
Region 1 $^{1}$ & 546 & 1075 & 1075 \\
Region 2 $^{1}$ & 5798 & 2652 & 2366 \\
Total $^{1}$ & 6344 & 3727 & 3442 \\
Relative time difference $^{2}$ & 1.84 & 1.08 & 1.00 \\
${ }^{1}$ Execution time in milliseconds. & &
\end{tabular}

\section{Multi-Objective Tuning of Multi-Region PROGRAMS}

In this section we firstly introduce some background related to multi-objective auto-tuning of programs. Afterwards, we state the main challenges when tuning multi-region programs.

\section{A. Background on Multi-Objective Auto-Tuning}

Auto-tuners may optimize several objectives which sometimes conflict with each other. This means that optimizing one of them is only possible by worsening the value of at least one of the other objectives. The mathematical solution to such problems is not defined by a single point, but by a set of points representing a trade-off between these objectives. The set of solutions representing the optimal trade-off between the considered objectives is known as Pareto set.

Our approach to apply multi-objective software auto-tuning consists in computing the Pareto set or an approximation of it [13]. Often, related work reduces multi-objective optimization problems to a single objective one by using fixed weights for the individual objectives. Therefore, they try to find a single solution which is near optimal in a pre-defined set of preferences for the objectives. Computing the Pareto set instead of a single solution is often incorrectly cited by related work with the belief that it implies a manual selection of a solution by the user which is just one possibility. However, different alternatives comprise a selection based on preferences specified a posteriori - i.e. once the Pareto set is computed. This latter approach does not require manual interaction of the user, and has the advantage that the computed solution belongs to the optimal trade-off. While computing the Pareto set may seem to require a higher computational effort than computing a single solution, literature in multi-objective optimization shows that this is not necessarily the case [14]. Indeed, the opposite is often true: computing the whole Pareto set may be easier than computing some individual solutions within it, as finding solutions which show a good compromise between two or more objectives implies a different way of navigating the search space. 


\section{B. Challenges in Tuning Multi-Region Programs}

We focus on programs composed of multiple regions. Tuning such programs requires to tune each of these regions. A region's performance may depend on the way other regions are executed, what data they access, and other side effects. Neighboring regions on a control flow are not independent of each other. This issue implies that regions should not be tuned in isolation, which has been observed in [2].

Tuning multi-region applications introduces additional challenges. Firstly, the search space of possible configurations of a program grows exponentially with the number of regions. For example, the matrix multiplication kernel considered in [12] consists of a single region. That region requires to tune three tiling dimensions and the number of threads. For a problem size of 14,000 - i.e. matrices of $14,000 \times 14,000$ elementsand a machine with 32 cores, the search space of possible program executions is $700^{3} \times 32 \simeq 10^{10}$. If a program consists of two regions similar to that one, the search space would be $\left(700^{\times} 32\right)^{2} \simeq 10^{20}$. Larger search spaces often reduce effectiveness of search methods [15]. Secondly, in a multiobjective multi-region scenario, it is crucial that the parameter values for the different regions within a program aim the optimization of the same objective. Otherwise, if two regions are assigned parameter values optimizing different objectives, most likely the execution of both regions together will not be optimal for any of these objectives. For example, this is the case when half of the regions within a program would be executed with optimal parameter values for a given objective and the other half of the regions with parameter values optimal for a conflicting objective.

Our goal in this paper is to design an auto-tuner that can find a single Pareto set of configurations for a given program with multiple regions. While the parameter values of every region are tuned separately, we measure the effect of changing the parameter values of a region regarding the entire program instead of considering the effect only for individual region executions. In this way, we optimize the whole program execution instead of focusing on specific regions. After the Pareto set for the whole program is computed, a single configuration for the entire program can be selected from the Pareto set, either manually or automatically. This approach differs from the one proposed in [12], which is based on computing an individual Pareto set for every single region in isolation, making this approach prone to the performance penalties described in Section VIII. Furthermore, computing a Pareto set independently for every region requires a decision making process for every single region. Therefore, the approach presented in [12] is unfeasible for tuning programs with a large number of regions. A feasible way to compare the approach in Jordan et al. [12] to the one presented in this paper is using the same set of parameters for every region of the program, thereby reducing the search space and producing only a single Pareto set for the entire program. Additionally, the auto-tuner can evaluate the performance of a configuration for all regions at once which makes it aware of eventual performance penalties caused by region interferences. In Section VI, we compare this version of the auto-tuner presented in [12], which we call RS-GDE3
Global, against the new version of this paper.

\section{Method}

Our approach extends the RS-GDE3 algorithm presented in [12], which is based on iterative compilation. It uses a fixed size set of different program configurations to be executed on the target architecture in order to determine their performance. RS-GDE3 refers to this set as population. Iterative compilation methods update this set across different iterations by generating possibly better performing configurations for the program being tuned. In the case of RS-GDE3, this is done by generating a new population called offspring population from the current population as explained later in this section. At the end of every iteration, the current population is updated by considering its content and the content of the offspring population. Details about how configurations are chosen to be part of the population for the next iteration can be found [12].

In order to tune multi-region programs regarding multiple objectives, we need to overcome the following problems:

1) All the parameter values within a configuration should aim for a common goal. If the tuner generates a program where a region $a$ uses parameter values optimized for a given objective, and for a subsequent region $b$ it uses the best parameter values regarding another objective, then the execution of both regions will unlikely be optimal for any of these objectives nor will it represent an optimal trade-off.

2) An intractable large search space, which may reduce the effectiveness of the search performed by RS-GDE3.

3) Existing or changing parameter settings of one region that may negatively impact other regions, introducing additional overheads.

4) Well performing sets of parameter values for individual regions may be discarded by the tuner if they are considered in combination with poorly performing parameter values for other regions.

To solve the first problem, our approach does not consider regions in isolation. Instead, our configurations are comprised of the parameter values of all regions and will be kept as part of the population only if they contribute to optimize the whole program.

To overcome the second problem, finding a good starting point in this huge search space is crucial to improve the effectiveness of the tuner. For this reason, we perform a global pre-tuning phase. During the pre-tuning phase, the auto-tuner uses the same set of parameter values for every region within the program. The idea is to reduce the size of the search space, making it easier for tuner to find the best configurations within the limited search space. We use these configurations as the starting point of a second tuning phase where every region can have a different set of parameter values. The global pretuning takes place during a few iterations at the beginning of our method. Besides reducing the dimensionality of the search space, the pre-tuning phase also helps to overcome the third problem, since the found configurations avoid overheads caused by changing hardware settings between regions. After 
1: for all configurations parent in population $P$ do

2: $\quad$ Randomly pick configurations $c_{1}, c_{2}$ and $c_{3}$ from $P$

3: $\quad$ diff $\leftarrow c_{3}+\left(c_{1}-c_{2}\right) / 2$

4: $\quad$ Create empty configuration $c_{n e w}$

5: for all parameter $\mathrm{x}$ do

6: $\quad$ Randomly choose $\mathrm{x}$ value from either diff or parent

7: $\quad$ Add $x$ value to the newly generated configuration $c_{\text {new }}$.

8: end for

9: $\quad$ Add $c_{n e w}$ to newly generated Population $P_{n e w}$

10: end forreturn $P_{\text {new }}$

Fig. 1: General Differential Evolution.

the global pre-tuning phase, overheads that arise from changing hardware settings may occur. However the auto-tuner will discard these configurations, unless the benefit of the different parameter values for each region outweighs the overheads caused by using different parameter values for individual regions.

To deal with the problem related to the fourth issue, we developed a novel approach which we call recombination. RS-GDE3 is a population based algorithm that generates new configurations by applying an operator called differential evolution [16]. Differential evolution is an approximation technique which also accounts for genetic algorithms or simulated annealing. We chose to use differential evolution because it has been empirically shown that it generally needs lower computational effort to produce higher quality results than other approximation algorithms [17]. A differential evolution algorithm generates new configurations by combining some existing ones as shown in Figure 1.

While generating new configurations by combining some existing ones may be beneficial, it also represents a drawback. For example, if the parent configuration $c_{1}$ contains the best possible parameter values $p_{1}$ for region $a$ and the newly generated configuration $c_{2}$ contains the best possible parameter values $p_{2}$ for region $b$, it would make sense to compose a third configuration $c_{3}$ that uses $p_{1}$ in region $a$ and $p_{2}$ in region $b$. However this is not possible in RS-GDE3 due to the way the differential evolution operator works. The recombination strategy solves this problem by generating new configurations using a different method every second iteration. This new generation method takes the parent configuration and the newly generated one and swaps several of their parameter values. In particular, the best set of parameter values for individual regions out of the parent and the newly generated configuration are preserved. This approach maximizes the chances of combining parameter values which perform well.

A comparison of a traditional, region-aware auto-tuner to the novel approach using recombination is shown in Figure 2. The former, depicted in Figure 2a, generates a new offspring population out of the current population using differential evolution in every iteration and selects the best performing configurations from the current population and the offspring population to form the population for the next step, as it is described in [12]. The latter, depicted in Figure 2b, uses the differential evolution only in every second iteration. In the other iterations, a new offspring population is generated by recombining the parameter values of the configurations in the current population and the offspring population. The selection of configurations which will form the population of the next step is unaltered compared to the traditional approach. In order to maximize the benefit of the recombination steps, we are using two different types of recombination, which are applied in an alternating fashion:

1) The first type compares each configuration which has been generated during the last iteration of the optimizer with its parent's configuration. Out of those two configurations (offspring and parent), for each region it selects the set of parameter values which results in better values, averaged over all objectives, and combines them to a new configuration for the entire program.

2) The second type selects one configuration for each objective from the offspring population as well as their corresponding parents' configurations. From the parameter values of those configurations, it constructs a new configuration for each objective, combining the sets of parameter values of the regions which deliver the best performance for the corresponding objective.

Finally, the pseudo-code of the whole method is given in Figure 3.

\section{IMPLEMENTATION}

The presented framework is implemented within the Insieme source-to-source compiler and runtime system presented in [12], based on branch inspire_l.3 which is freely available at [18]. The Insieme compiler performs, among other tasks, code analysis and code transformations. Insieme uses INSPIRE [19] as intermediate representation for extracting individual regions and applying the required code transformations. The transformed INSPIRE code is then converted back to C. The resulting source code is compiled to binary using the Gnu Gcc Compiler. For all measurements presented in this paper, we set the -03 flag to obtain the highest performance. In the case of transformations such as tiling, the Insieme compiler generates a different version for each tile size to be evaluated in order to obtain the best performance. This means, that a separate version of the code has to be generated and compiled for each configuration that needs to be evaluated.

The Insieme runtime system executes the transformed input code and measures its performance. The measurements are reported to the Insieme compiler which provides them to the auto-tuner. The Insieme runtime system allows to set the number of threads individually for each region and is also responsible for mapping the executed program to OS-level threads. For each OS-level thread used, one worker is created and started [20]. The number of workers created for a specific execution is equal to the maximum number of threads used by any region of the given program. Each worker is fixed to a specific CPU core in ascending order. When a region is executed with fewer threads than the number of workers started, the additional workers are send to sleep by the runtime system. 


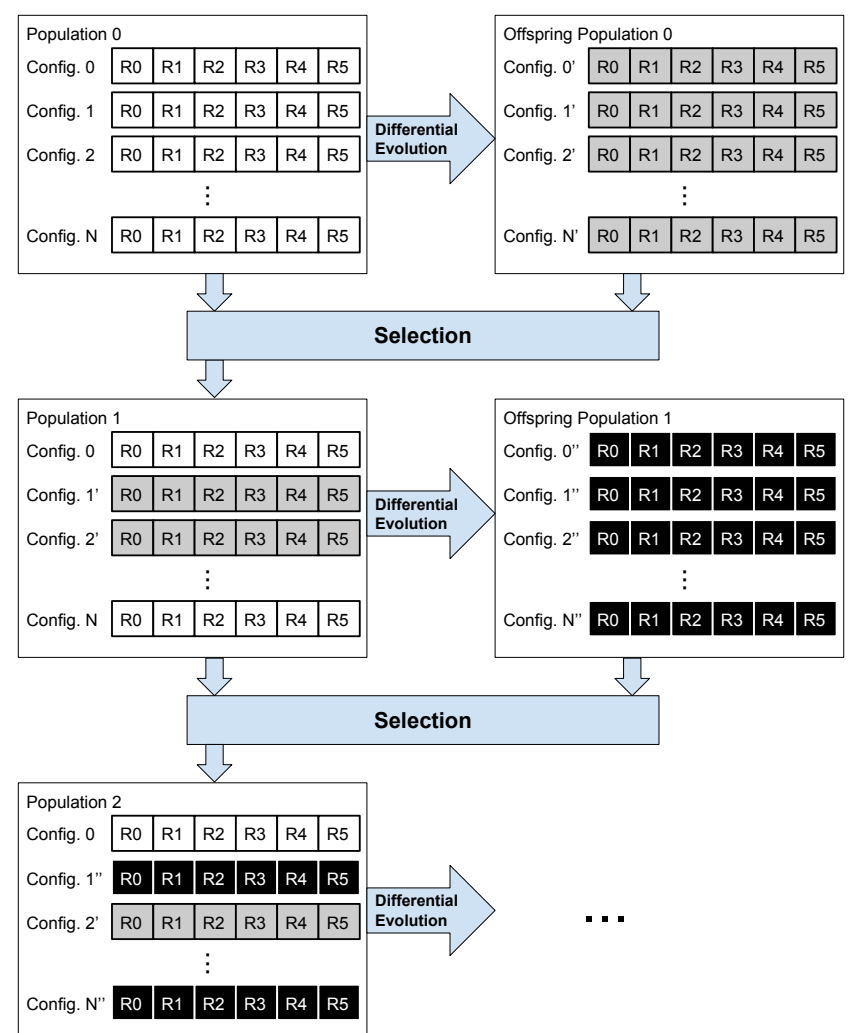

(a) Region-aware auto-tuner using GDE3 only. In each iteration an offspring population of $\mathrm{N}$ elements is generated out of the current population.

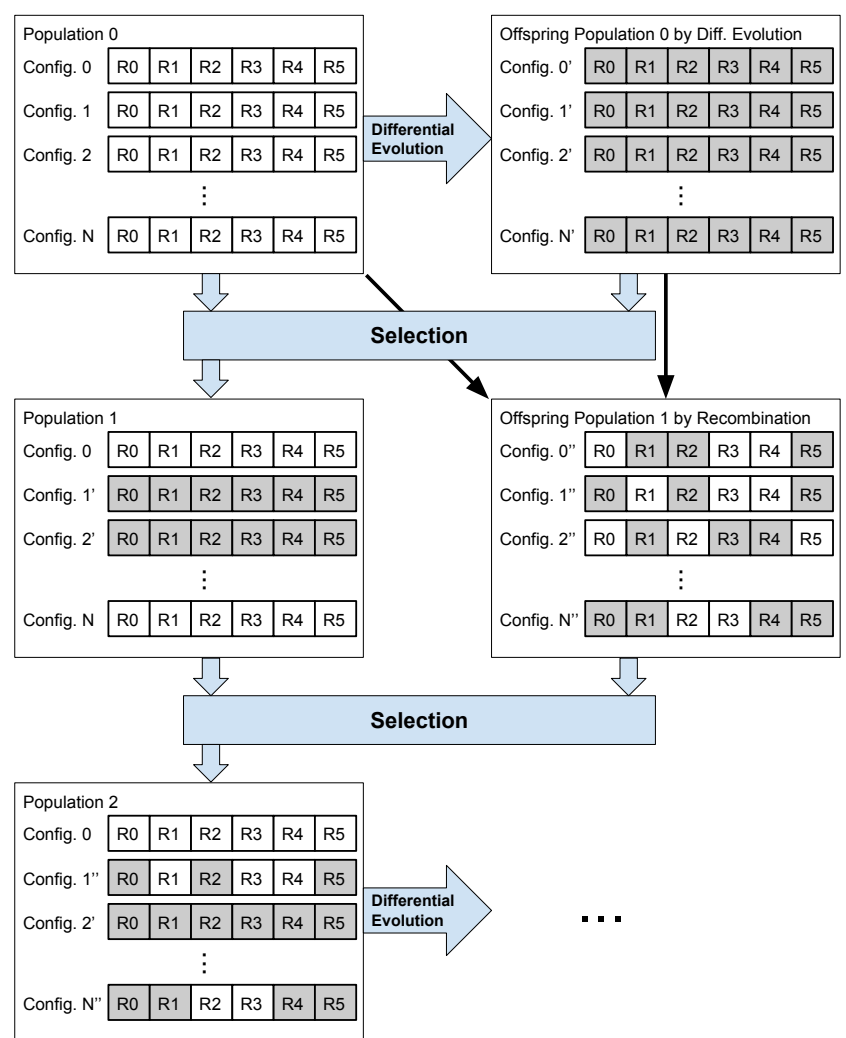

(b) Region-aware auto-tuner using GDE3 and recombination. In every second iteration, an offspring population is created by recombining the best performing settings for each individual region from the previous population and offspring population.

Fig. 2: Examples for the evolution of two region-aware auto-tuners over two iterations, using a population of $\mathrm{N}$ configurations, tuning a program with six regions (R0 to R5). Both auto-tuners perform $2 \times \mathrm{N}$ evaluations in this example. In each iteration, a new population is created by selecting the best configurations from the population of the previous iteration and the corresponding offspring population.

1: Generate a population $P_{\text {pre }}$ of configurations, where regions within the same configuration have the same parameter values

2: Set the iteration counter to zero

3: while iteration counter $\leq$ threshold do

4: $\quad$ Generate new offspring population $O P_{\text {pre }}$ using the differential evolution method

5: $\quad$ Update $P_{\text {pre }}$ with the best trade-off solutions from $P_{\text {pre }} \cup O P_{\text {pre }}$

6: $\quad$ Increase the iteration counter

7: end while

8: Generate a new population $P$ of configurations using solutions in $P_{\text {pre }}$, replicating the global set of parameter values for each region.

9: while iteration counter $\leq$ maximum number of iterations do

10: if iteration counter is even then

11: Generate an offspring population $O P$ using the differential evolution method

12: $\quad$ else

13: $\quad$ Generate an offspring $O P$ population using the recombination method

14: $\quad$ end if

15: Increase the iteration counter

16: $\quad$ Update $\mathrm{P}$ with the best trade-off solutions from $P \cup O P$

17: end while

18: return the non dominated solutions (i.e. the Pareto set) of $P$

Fig. 3: Multi-region auto-tuner using a combination of general evolution and recombination. 
The resource usage is calculated by multiplying the number of threads used with the execution time of each individual region. To measure the energy consumption we use Intel's RAPL interface, that has been shown to provide sufficiently accurate in-band energy measurements for modern Intel CPUs without the need of external energy measurement devices [21].

\section{A. Regions}

Our auto-tuner targets parallel programs implemented in C using OpenMP [22] for parallelization. As mentioned in Section I, the programs are subdivided into several regions. We define each parallel OpenMP for-loop to be a separate region for several reasons: Besides being parallel, these loops usually contain most of a program's computational work. Furthermore, the implicit synchronization following each parallel OpenMP for-loop is well suited as a point to vary the number of threads, while the number of threads cannot be changed within the body of an OpenMP for-loop. Additionally, the restrictions enforced by OpenMP on parallel for-loops, such as no continue, break or return statements as well as no modification of the iterator variable inside the loop's body, increase the probability that a loop nest starting with a parallel OpenMP for-loop is suitable for tiling. If an OpenMP loop is called several times from different contexts, each context creates a different region that can be tuned individually.

Loop nests which can be tiled are of special interest to us, as they typically have high optimization potential and consume most resources. Our auto-tuner examines whether a loop nest is suitable for tiling by using the Polyhedral Model [23] which is integrated in the Insieme Compiler. This analysis determines whether a loop nest is tilable and also provides information about the minimum and maximum tile size for this transformation.

For every region the auto-tuner can tune the number of threads that are used to execute it. Furthermore, for regions which are tilable, the tile size in each dimension is tuned.

\section{Testing Methodology}

To compare different approaches, we use the same objectives as in [12]. For the result $S$ of every auto-tuning run we calculate $|S|$ and $V(S) . S$ corresponds to the resulting Pareto set of the auto-tuner while $|S|$ is the number of elements in the Pareto set $S$. A larger number of elements of the Pareto set is considered superior, as it offers more flexibility to choose a desired solution. $V(S)$ defines the normalized size of the hypervolume covered by the performance measurements of the elements in the Pareto set $S$ [24], i.e. the relative size of a hypervolume formed by all points dominated by the points in $S$ in a normalized hyperrectangle defined by the highest and lowest measurement in each objective. When the function $V(S)$ is used to compare several solutions $S_{i}$ where $i \in[0, I]$ and $I \in \mathbb{N}^{+}$, this hyperrectangle is defined by the highest respectively lowest measurement in each objective of the combined Pareto set of all solutions $S_{i}$. This means, if all configurations in $S_{i}$ are dominated by configurations found in $S_{j}$ with $j \in[0, I]$ and $j \neq i$, the coverage $V\left(S_{i}\right)$ is 0 . A configuration $c_{0}$ dominates another configuration $c_{1}$ if $c_{0}$ delivers better performance than $c_{1}$ in every objective. As the coverage is calculated on a normalized hypervolume, the result of $V(S)$ ranges from 0 to 1 where 1 corresponds to an ideal solution covering the entire hypervolume, i.e. dominating all other solutions. For each approach we report the average population size $\overline{|S|}$ and hypervolume $\overline{V(S)}$ over 16 runs.

The huge search space of the tested programs prevents a comparison of the results of an auto-tuner to the theoretical optimum, as finding the theoretical optimum implies an exhaustive search over the entire search space.

\section{EXPERIMENTAL RESULTS}

This section presents the performance that we obtained with our approach on some exemplary test cases. The experiments are executed on two different machines. The first machine features two Intel Xeon E5-2690 v2 CPUs in a dual socket layout that are based on the Ivy Bridge-EP architecture with 20 cores in total. The second machine is equipped with four Intel Xeon E5-4650 CPUs, based on the Sandy Bridge-EP architecture and featuring a total of 32 cores. Hyperthreading has been disabled on both architectures. The clock frequency was fixed to the highest base frequency throughout all experiments.

We present the results obtained by different auto-tuners including random, global tuners and region-aware ones. The complete list is:

- Random: It randomly generates 3000 configurations with individual settings for each region.

- RS-GDE3 Global: It uses the RS-GDE3 tuner introduced in [12] to determine values for all tunable parameters. This version resembles is a version of the auto-tuner presented in [12] as described in Section III-B. Every region within the entire program uses the same set of parameter values, thereby reducing the search space. Solutions are generated with the differential evolution operator described in [12].

- RS-GDE3 Region: Region-aware version of RS-GDE3 Global, which sets the parameter values for every region individually.

- RS-GDE3 Region GPT: Extends the RS-GDE3 Region using a global pre-tuning phase as described in Section III. The first ten iterations are devoted to this phase.

- RS-GDE3 Recombination: Based on RS-GDE3 Region, but new configurations are generated using the recombination method described in Section III in every second iteration.

- RS-GDE3 Recombination GPT: RS-GDE3 Recombination with a global pre-tuning phase that uses ten iterations.

All compared RS-GDE3 auto-tuners variations use a population size of 30 and perform 100 iterations, leading to a total of about 3000 executions of the program (as performed also by the Random tuner). Our experiments did not show any significant performance improvements by enlarging the population any further with that budget of iterations.

The effectiveness of these approaches are evaluated on three benchmarks. Two of them, $\mathrm{mg}$ and bt, are taken from the NAS parallel benchmarks [25] C/OpenMP implementation by 
the Omni group [26]. Bt is a block tri-diagonal solver for nonlinear partial differential equations while mg approximates the solution to a three-dimensional discrete Poisson equation using a multi-grid method. For bt we choose problem size $w$, for $\mathrm{mg}$ the problem size $b$, in order to get reasonable execution times for auto-tuning. The heated-plate benchmark [27] is a stencil-code solving the steady heat equation on a two dimensional, rectangular plate. The matrix size used for this benchmark was set to $384 \times 384$ elements. The total number of regions, the number of regions to which tiling can be applied as well as the total number of tunable parameters for each of those programs are listed in Table II. This Table also indicates the search space size when tuning those programs. The number of tunable parameters is the sum of the number of tiling dimensions of all regions plus the number of regions, as we can set the number of threads separately for each region. The search space is the product of the ranges for each of those parameters. Therefore, the size of the search space depends on the target architecture, as a higher number of cores also provides more tuning possibilities. For each tuned program, we include the results of comparing the six previously described auto-tuners in Table III. The computed Pareto set of most autotuners contains 30 elements, i.e. the entire population. The RSGDE3 Recombination GPT auto-tuner delivers the best result in terms of hypervolume in all cases. The global pre-tuning phase yields a higher improvement on the Sandy Bridge-EP architecture than on the Ivy Bridge-EP which can be explained by their differing socket numbers: Whereas the Ivy Bridge-EP system has only two sockets, the Sandy Bridge-EP system has four sockets. Changing the number of threads between regions can imply an additional cache coherency overhead when the regions are executed in different number of sockets. This overhead is a consequence of not having shared cache between sockets. Therefore, it is beneficial to start with a configuration that does not change the number of threads between regions, which is achieved by the global pre-tuning phase.

TABLE II: Search space description for the evaluated programs.

\begin{tabular}{l|ccc} 
& $\mathrm{mg}$ & heated-plate & $\mathrm{bt}$ \\
\hline \#Regions & 94 & 10 & 122 \\
\#Tilable Regions & 82 & 4 & 114 \\
\#Tunable Parameters & 268 & 18 & 453 \\
Search Space Size & $10^{590}$ & $10^{33}$ & $10^{897}$ \\
Search Space Size $^{2}$ & $10^{609}$ & $10^{35}$ & $10^{922}$ \\
${ }^{1}$ On Ivy Bridge-EP & & & \\
${ }^{2}$ On Sandy Bridge-EP & & &
\end{tabular}

The time required for the auto-tuning is dominated by the time needed to compile and execute the program. Therefore, shorter and faster programs can be tuned in less time. The tuning time for our test cases is shown in Table IV. The Random auto-tuner exhibits the longest tuning time, as it typically evaluates the configurations with the lowest performance. The fastest auto-tuner over all test cases is the RSGDE3 Global, because it never experiences any slowdowns from performance penalties caused by region interferences. From the region-aware auto-tuners, those without a global pretuning phase are slower than the auto-tuners with a global pre-tuning phase in most cases. The latter converge faster to a population with reasonably fast configurations, which leads to significantly lower execution times of the tuned program. Similarly, the region-aware auto-tuners using the Recombination step are faster than their counterparts using only the traditional differential evolution in most cases, as the average execution time of the resulting program versions is shorter.

In addition to the Pareto set size $|S|$ and hypervolume $V(S)$ we also report the objective values of the best configuration found by the RS-GDE3 Recombination GPT auto-tuner for the three real world codes compared to the non-optimized (without auto-tuning) versions. To calculate the speedup we use the best configuration from the auto-tuner's Pareto set for each individual objective. Typically, this is a different configuration for every objective. We compare these configurations to two non-optimized configurations that do not apply any tiling: the sequential version, using only one thread and the parallel version using all threads available on the corresponding machine. The results shown in Table $\mathrm{V}$ demonstrate the superior performance compared to the non-optimized versions, both sequential and parallel, in every objective. As expected, the largest improvement over the sequential version can be achieved in wall time (up to 13.8 fold) while the parallel version is primarily outplayed in resource usage. Especially on the Sandy Bridge-EP architecture with 32 cores, the nonoptimized version suffers from the moderate scalability of heated-plate and bt, allowing our auto-tuner to achieve an improvement factor of up to 63.7 in resources usage. These results clearly demonstrate the benefit of our region-aware multi-objective auto-tuner, even if only a single objective is of interest. This is underlined by the achieved speedup of the auto-tuner over the parallel version, which ranges from 1.3 to 4.0 on the tested architectures and programs. If a well-balanced trade-off solution across several objectives is required, the benefit may be even higher, depending on the user's preferences.

\section{DISCUSSION}

The results presented in the previous section demonstrate that the best version of our region-aware auto-tuner, the RSGDE3 Recombination GPT, outperforms a global auto-tuner based on RS-GDE3 (called RS-GDE3 Global in this paper).

When comparing the Pareto sets generated by these two tuners, we observe that some configurations in the Pareto set of the RS-GDE3 Global auto-tuner are dominated by others in the Pareto set of the RS-GDE3 Recombination GPT. This means that there are configurations computed by the global tuner which are worse in all the considered objectives than some of the configurations computed by the region-aware tuner.

Next, we analyze the configurations computed by these two algorithms in order understand how region-aware tuners exploit different parameter values in different regions of the same program. To this end, we compare the obtained results on two of the considered applications, the bt and the heated-plate. For these comparisons, we pick a configuration computed by each of these two tuners and observe the parameter values within each region. 
TABLE III: Results of several auto-tuner variants for different benchmarks on two different architectures.

\begin{tabular}{|c|c|c|c|c|c|c|c|c|c|c|c|c|}
\hline & & ng & \multicolumn{2}{|c|}{$\begin{array}{l}\text { Ivy Bridge-EP } \\
\text { heated-plate }\end{array}$} & \multicolumn{2}{|c|}{ bt } & \multicolumn{2}{|c|}{$\mathrm{mg}$} & \multicolumn{2}{|c|}{$\begin{array}{c}\text { Sandy Bridge-EP } \\
\text { heated-plate }\end{array}$} & \\
\hline & $\overline{|S|}$ & $\overline{V(S)}$ & $\overline{|S|}$ & $\overline{V(S)}$ & $\overline{|S|}$ & $\overline{V(S)}$ & $\overline{|S|}$ & $\overline{V(S)}$ & $\overline{|S|}$ & $\overline{V(S)}$ & $\overline{|S|}$ & $\overline{V(S)}$ \\
\hline $\operatorname{Ran}$ & 14.9 & 0.0721 & 12.0 & 0.0284 & 26.9 & 0 & 26.2 & 0 & 4.3 & 0 & 12.4 & 0 \\
\hline RS-GDE3 Global & 29.9 & 0.9058 & 29.1 & 0.6475 & 23.8 & 0.6399 & 27.6 & 0.8572 & 29.9 & 0.7802 & 23.6 & 0.6148 \\
\hline RS-GDE3 Region & 29.9 & 0.3352 & 29.4 & 0.6441 & 29.8 & 0 & 30.0 & 0 & 29.9 & 0.7543 & 23.1 & 0 \\
\hline RS-GDE3 Region GP & 30.0 & 0.9013 & 30.0 & 0.6699 & 30.0 & 0.6122 & 30.0 & 0.8570 & 29.9 & 0.7975 & 30.0 & 0.6016 \\
\hline & 30.0 & 0.8 & 30. & & 30.0 & 0.6 & 30.0 & 0.0560 & 29.2 & & 30.0 & 0.5767 \\
\hline RS-GDE3 Recombination & 30.0 & 0.9168 & 30.0 & 0.6857 & 30.0 & 0.7058 & 30.0 & 0.8836 & 29.6 & 0.8199 & 30.0 & 0.7101 \\
\hline
\end{tabular}

TABLE IV: Tuning time in seconds of several auto-tuner variants for different benchmarks on two different architectures.

\begin{tabular}{l|ccc|ccc} 
& \multicolumn{3}{|c|}{ Ivy Bridge-EP } & \multicolumn{3}{c}{ Sandy Bridge-EP } \\
& $\mathrm{mg}$ & heated-plate & $\mathrm{bt}$ & $\mathrm{mg}$ & heated-plate & $\mathrm{bt}$ \\
\hline Random & 21945 & 26638 & 24242 & 31882 & 34774 & 38621 \\
RS-GDE3 Global & 16262 & 9605 & 15218 & 19480 & 15125 & 27274 \\
RS-GDE3 Region & 16903 & 12829 & 19187 & 33273 & 20596 & 42808 \\
RS-GDE3 Region GPT & 19318 & 10784 & 16639 & 21371 & 17771 & 28754 \\
RS-GDE3 Recombination & 19868 & 10839 & 18758 & 31164 & 15006 & 34038 \\
RS-GDE3 Recombination GPT & 19732 & 10257 & 18116 & 22686 & 16217 & 32209
\end{tabular}

TABLE V: Improvement over non-optimized versions i.e. not tiled with a constant number of threads, in each individual metric achieved by the RS-GDE3 Recombination GPT auto-tuner.

\begin{tabular}{|c|c|c|c|c|c|c|c|c|c|c|c|c|}
\hline & \multicolumn{4}{|c|}{$\begin{array}{l}\text { Ivy Bridge-EP } \\
\text { heated-plate }\end{array}$} & \multicolumn{2}{|c|}{ bt } & \multicolumn{6}{|c|}{ Sandy Bridge-EP } \\
\hline & $\mathrm{s}^{1}$ & $\mathrm{p}^{2}$ & $s^{1}$ & $\mathrm{p}^{2}$ & $\mathrm{~s}^{1}$ & $\mathrm{p}^{2}$ & $\mathrm{~s}^{1}$ & $\mathrm{p}^{2}$ & $\mathrm{~s}^{1}$ & $\mathrm{p}^{2}$ & $\mathrm{~s}^{1}$ & $\mathrm{p}^{2}$ \\
\hline wall time & 7.7 & 1.3 & 13.7 & 4.0 & 4.4 & 3.2 & 3.7 & 2.2 & 7.6 & 4.0 & 2.4 & 4.0 \\
\hline energy & 3.2 & 2.4 & 8.1 & 4.5 & 2.1 & 3.6 & 2.2 & 6.0 & 4.5 & 7.5 & 1.6 & 10.6 \\
\hline resource usage & 1.4 & 4.8 & 3.4 & 20.2 & 1.5 & 21.9 & 1.3 & 24.4 & 2.7 & 46.4 & 1.2 & 63.7 \\
\hline
\end{tabular}

For the first comparison, the two configurations are taken from the Pareto set generated by the two auto-tuners on the Sandy Bridge-EP architecture. We refer to the configuration computed by the region-aware tuner as $C_{r}$, and to the configuration computed by the global tuner as $C_{g}$. The first thing to note is that the tiling values used for different regions in $C_{r}$ show a high variation. For example, while region 40 is tiled using the values $\{368,13,1\}$, the region 88 uses the tiling parameters $\{1,1,2\}$. The tile sizes used by $C_{g}$ are $\{1,8,2\}$ for all the regions within the program. All regions in $C_{g}$ are executed with eight threads, which corresponds to the maximum number of cores on one socket on our Sandy Bridge-EP architecture. Also in $C_{r}$, the maximum number of threads used is eight, meaning that some of the regions are also executed using eight cores. However, many regions in $C_{r}$ are executed with fewer threads, where any number between one and eight is used at least once. This results in the objective values presented Table VI. While in terms of wall time and energy consumption both configurations are similar, the resource usage of $C_{g}$ is $20 \%$ higher than for $C_{r}$. This means, $C_{r}$ is as fast and consumes as little energy as $C_{g}$ using less resources. This is possible because the region-aware tuner found a configuration that executes regions which do scale well up to eight threads with such number of threads; at the same time, regions that do not benefit from being executed on eight cores are executed with fewer threads. Such a degree of adaption is not possible with any auto-tuner that uses the same parameter values for every region in the entire program.

For the second comparison, we present the performance figures for two configurations for the heated-plate benchmark
TABLE VI: Performance of two individual configurations in bt on the Sandy Bridge-EP architecture.

\begin{tabular}{l|ccc} 
& $C_{g}{ }^{1}$ & $C_{r}{ }^{2}$ & Improvement \\
\hline wall time (ms) & 2076 & 2074 & 1.00 \\
energy consumption (J) & 148 & 147 & 1.00 \\
resource usage (ms) & 16611 & 13838 & 1.20 \\
1 Taken out of the Pareto set generated with the RS-GDE3 \\
Global auto-tuner. \\
2 Taken out of the Pareto set generated with the RS-GDE3 \\
Recombination GPT auto-tuner.
\end{tabular}

on the Ivy Bridge-EP architecture. Again we label $C_{r}$ the configuration found by the region-aware tuner and $C_{g}$ the configuration found by the global tuner. In this case, for $C_{g}$ we choose the configuration with the least resources usage from the Pareto set. While $C_{r}$ is not the configuration leading to the lowest resource usage in its Pareto set, it still dominates $C_{g}$. Obviously, $C_{g}$ uses only one thread for every region in order to minimize the resource usage and the tile sizes used by this configuration are $\{1,214\}$. In contrast to that, $C_{r}$ uses two threads to execute the biggest region of heated-plate, i.e. region 8. The increased number of threads also requires a different tile size in order to perform well; in this case it uses $\{31,251\}$. All other regions, which account for more than $1 \%$ of the total wall time, use very similar parameter values as the one used in $C_{g}$ : they are executed using only one thread, and the tile size in the first dimension is equal to 1 , while the tile size in the second dimension varies from 233 to 251 . This indicates, that a tile size of 1 in the first dimension is beneficial when regions are executed sequentially, while a higher number of threads 
benefits from larger tile sizes. The combination of a custom tile size and higher number of threads for region 8 of heated-plate results in a significantly lower wall time as shown in Table VII. As that region does scale well, $C_{r}$ has also a slight advantage over $C_{g}$ in both, resource usage and energy consumption, despite the increased number of cores used. Additionally, as indicated in the table, the rather different parameter values for region 8 cause a $25 \%$ drop in wall time, compared to $C_{g}$. As in the comparison before, these performance figures can only be achieved using different parameter values for the individual regions of the program.

TABLE VII: Performance of two individual configurations in heated-plate on the Ivy Bridge-EP architecture.

\begin{tabular}{l|ccc} 
& $C_{g}{ }^{1}$ & $C_{r}{ }^{2}$ & Improvement \\
\hline wall time (ms) & 2323 & 1749 & 1.33 \\
energy consumption (J) & 65 & 63 & 1.04 \\
resource usage (ms) & 2323 & 2289 & 1.02 \\
1 Taken out of the Pareto set generated with the RS-GDE3 \\
Global auto-tuner. \\
2 Taken out of the Pareto set generated with the RS-GDE3 \\
Recombination GPT auto-tuner.
\end{tabular}

\section{RELATED WORK}

In the literature we find several frameworks for software auto-tuning, for example self-tuning libraries like ATLAS [3], OSKI [4], SPIRAL [5] or FFTW [6], or other auto-tuning frameworks including Active Harmony [7], Sequoia [28], PetaBricks [8], [9], Patus [10], and OpenTuner [11].

In the past, most auto-tuners focused on improving the wall time of programs. However, recent work shows an inarguable attention to tune applications regarding several objectives. Besides wall time, energy consumption entailed by a program's execution is becoming a popular objective [29], [30], [31], [32], [33], [34], [35], [2], [36]. Resource usage [12], [36], compilation time, or the size of the executable binary [37], [38], [39] also received attention in related work. Most of these works fail to capture the trade-off between these objectives and reduce them to a single one. Only a few works focus on computing and analyzing the trade-off between several conflicting objectives [40], [12], [41].

All the aforementioned works applied the same tuning options to the whole program. Approaches which individually tune code regions and examine their inter-relationships with respect to single or multiple objectives are rare. A major issue is the definition of code regions for programs. In [42] program functions are considered to be the regions to tune. In MPI programs regions are often defined as the code between pairs of communication directives in [2]. In [43] regions are obtained from applications Regions within the same cluster are tuned using the same parameters or code transformations. The Periscope tool of the AutoTune project ${ }^{1}$ tunes regions regarding any function measuring properties of that function (run-time, energy consumed, etc.). Although different objectives can be tuned, they are not considered simultaneously. Furthermore, Periscope tunes regions individually without considering side effects among regions. In contrast to the

${ }^{1}$ http://www.autotune-project.eu/ framework presented in this work, Periscope does not describe a methodology to identify regions within a program.

In [42], [43], programs are split into several regions which are tuned in isolation. However, the authors of [2] show that regions within a program impact each others execution time behavior. They demonstrate that when regions are executed with the best set of parameter values known so far, a nonnegligible penalty may be paid as a result of changing hardware settings across adjacent regions. The same work also discusses the benefits of using the same set of parameter values for every region in the entire program versus a per-region tuning approach, and the need for tuning mechanisms that can find configurations aware of interferences between regions.

\section{CONCLUSiON}

Most existing work on auto-tuning focuses on a global setting of parameter values which are fixed for the entire program, ignoring the optimization potential by customizing parameter values to individual region's peculiarities. In this paper, we introduced a novel auto-tuning framework that is based on a source-to-source compiler, a runtime system and a new RS-GDE3 auto-tuner variation to provide a solution for multi-region, multi-objective auto-tuning. The challenges introduced by the huge search space, region dependencies and conflicting objectives are tackled by adding a pre-tuning phase to the region-aware auto-tuner which tunes the program using the same parameter values for all regions, as well as an intermediate evolutionary step for the RS-GDE3 autotuner, that generates new configurations by recombining the parameter values generated in previous steps.

Experiments have shown that our new approach is more effective in tuning three different programs on two different parallel computers than non-region-aware global auto-tuning. We outperform a non-region-aware RS-GDE3 auto-tuner in hypervolume $V(S)$ by up to $15 \%$. Furthermore, we demonstrated that our approach reaches up to 7.6, 10.5 and 61.6 fold improvements in wall time, energy consumption and resource usage respectively, over the non-optimized parallel version.

\section{ACKNOWLEDGEMENTS}

This project has received funding from the European Unions Horizon 2020 research and innovation programme as part of the FETHPC AllScale project under grant agreement No 671603.

\section{REFERENCES}

[1] K. Naono, K. Teranishi, J. Cavazos, and R. Suda, Software Automatic Tuning (From Concepts to State-of-the-Art Results). Secaucus, NJ, USA: Springer-Verlag New York, Inc., 2010.

[2] D. Li, B. R. de Supinski, M. Schulz, D. S. Nikolopoulos, and K. W. Cameron, "Strategies for energy-efficient resource management of hybrid programming models." IEEE Trans. Parallel Distrib. Syst., vol. 24, no. 1, pp. 144-157, 2013. [Online]. Available: http://dblp.uni-trier.de/db/journals/tpds/tpds24.html \\#LiSSNC13

[3] R. Whaley and J. Dongarra, "Automatically tuned linear algebra software," in Proceedings of the 1998 ACM/IEEE conference on Supercomputing (CDROM). IEEE Computer Society, 1998, pp. 1-27.

[4] R. Vuduc, J. Demmel, and K. Yelick, "Oski: A library of automatically tuned sparse matrix kernels," in Journal of Physics: Conference Series, vol. 16. IOP Publishing, 2005, p. 521. 
[5] M. Puschel, J. Moura, J. Johnson, D. Padua, M. Veloso, B. Singer, J. Xiong, F. Franchetti, A. Gacic, Y. Voronenko et al., "Spiral: Code generation for dsp transforms," Proceedings of the IEEE, vol. 93, no. 2, pp. 232-275, 2005

[6] M. Frigo, "A fast fourier transform compiler," in Acm Sigplan Notices, vol. 34, no. 5. ACM, 1999, pp. 169-180.

[7] C. Tapus, I. Chung, and J. Hollingsworth, "Active harmony: Towards automated performance tuning," in Supercomputing, ACM/IEEE 2002 Conference. IEEE, 2002, pp. 44-44.

[8] J. Ansel, C. Chan, Y. Wong, M. Olszewski, Q. Zhao, A. Edelman, and S. Amarasinghe, PetaBricks: a language and compiler for algorithmic choice. ACM, 2009, vol. 44, no. 6.

[9] S. P. Amarasinghe, "Petabricks: a language and compiler based on autotuning," in HiPEAC, 2011, p. 3.

[10] M. Christen, O. Schenk, and H. Burkhart, "Patus: A code generation and autotuning framework for parallel iterative stencil computations on modern microarchitectures," in Proceedings of the 2011 IEEE International Parallel \& Distributed Processing Symposium, ser. IPDPS '11. Washington, DC, USA: IEEE Computer Society, 2011, pp 676-687. [Online]. Available: http://dx.doi.org/10.1109/IPDPS.2011.70

[11] J. Ansel, S. Kamil, K. Veeramachaneni, J. Ragan-Kelley, J. Bosboom, U.-M. O'Reilly, and S. Amarasinghe, "Opentuner: An extensible framework for program autotuning," in International Conference on Parallel Architectures and Compilation Techniques, Edmonton, Canada, August 2014. [Online]. Available: http://groups.csail.mit.edu/commit/ papers/2014/ansel-pact14-opentuner.pdf

[12] H. Jordan, P. Thoman, J. J. Durillo, S. Pellegrini, P. Gschwandtner, T. Fahringer, and H. Moritsch, "A multi-objective auto-tuning framework for parallel codes," in Proceedings of the International Conference on High Performance Computing, Networking, Storage and Analysis, ser. SC '12. Los Alamitos, CA, USA: IEEE Computer Society Press, 2012, pp. 10:1-10:12. [Online]. Available: http://dl.acm.org/citation.cfm?id=2388996.2389010

[13] C. Coello, D. Van Veldhuizen, and G. Lamont, Evolutionary Algorithms for Solving Multi-Objective Problems, ser. Genetic Algorithms and Evolutionary Computation. Springer US, 2013. [Online]. Available: https://books.google.de/books?id=VmnTBwAAQBAJ

[14] J. Handl, S. C. Lovell, and J. Knowles, "Multiobjectivization by decomposition of scalar cost functions," in Proceedings of the International Conference on Parallel Problem Solving from Nature, 2008, 2008.

[15] J. J. Durillo, A. J. Nebro, C. A. C. Coello, J. Garcia-Nieto, F. Luna, and E. Alba, "A study of multiobjective metaheuristics when solving parameter scalable problems," IEEE Transactions on Evolutionary Computation, vol. 14, no. 4, pp. 618-635, Aug 2010.

[16] R. Storn and K. Price, "Differential evolution \&ndash; a simple and efficient heuristic for global optimization over continuous spaces," $J$. of Global Optimization, vol. 11, no. 4, pp. 341-359, Dec. 1997. [Online]. Available: http://dx.doi.org/10.1023/A:1008202821328

[17] J. Durillo, A. Nebro, F. Luna, C. Coello Coello, and E. Alba, "Convergence speed in multi-objective metaheuristics: Efficiency criteria and empirical study," International Journal for Numerical Methods in Engineering, vol. 84, no. 11, pp. 1344-1375, 2010.

[18] "Insieme Source Code Repository," https://github.com/insieme/insieme/ tree/inspire $\backslash \_1.3,2017$.

[19] H. Jordan, "Insieme: A compiler infrastructure for parallel programs," $\mathrm{Ph} . \mathrm{D}$. dissertation, University of Innsbruck, 82014.

[20] P. Thoman, "Insieme-rs: A compiler-supported parallel runtime system," Ph.D. dissertation, University of Innsbruck, 72013.

[21] M. Hähnel, B. Döbel, M. Völp, and H. Härtig, "Measuring energy consumption for short code paths using rapl," ACM SIGMETRICS Performance Evaluation Review, vol. 40, no. 3, pp. 13-17, 2012

[22] OpenMP Architecture Review Board, "OpenMP Application Program Interface," http://www.openmp.org/mp-documents/OpenMP3.1. pdf, 2015.

[23] P. Feautrier and C. Lengauer, Encyclopedia of Parallel Computing. Springer, 2011, ch. Polyhedron Model, pp. 1581-1592.

[24] E. Zitzler and L. Thiele, "Multiobjective evolutionary algorithms: A comparative case study and the strength pareto approach," Trans. Evol. Comp, vol. 3, no. 4, pp. 257-271, Nov. 1999. [Online]. Available: http://dx.doi.org/10.1109/4235.797969

[25] NASA, "The NAS Parallel Benchmarks," http://www.nas.nasa.gov/ Software/NPB/, 2016

[26] "Omni OpenMP Compiler website," http://www.hpcs.cs.tsukuba.ac.jp/ omni-compiler/download/download-benchmarks.html, 2016.

[27] J. Burkardt, "HEATED_PLATE_OPENMP," http://people.sc.fsu.edu/ $\sim$ jburkardt/c_src/heated_plate_openmp/heated_plate_openmp.html, Mar. 2016
[28] K. Fatahalian, T. Knight, M. Houston, M. Erez, D. Horn, L. Leem, J. Park, M. Ren, A. Aiken, W. Dally et al., "Sequoia: programming the memory hierarchy," in SC 2006 Conference, Proceedings of the ACM/IEEE. IEEE, 2006, pp. 4-4.

[29] J. H. Laros, III, K. T. Pedretti, S. M. Kelly, W. Shu, and C. T. Vaughan, "Energy based performance tuning for large scale high performance computing systems," in Proceedings of the 2012 Symposium on High Performance Computing, ser. HPC '12. San Diego, CA, USA: Society for Computer Simulation International, 2012, pp. 6:1-6:10. [Online]. Available: http://dl.acm.org/citation.cfm?id=2338816.2338822

[30] M. Rahman, L.-N. Pouchet, and P. Sadayappan, "Neural network assisted tile size selection," in International Workshop on Automatic Performance Tuning (IWAPT'2010). Berkeley, CA: Springer Verlag, Jun. 2010.

[31] A. Tiwari, M. Laurenzano, L. Carrington et al., "Auto-tuning for energy usage in scientific applications," in Euro-Par 2011: Parallel Processing Workshops. Springer, 2012.

[32] S. Rahman, J. Guo, A. Bhat et al., "Studying the impact of applicationlevel optimizations on the power consumption of multi-core architectures," in Proc. of the 9th conference on Computing Frontiers. ACM, 2012.

[33] R. Springer, D. Lowenthal, B. Rountree et al., "Minimizing execution time in mpi programs on an energy-constrained, power-scalable cluster," in Proc. of the eleventh ACM SIGPLAN PPoPP. ACM, 2006.

[34] J. Peraza, A. Tiwari, M. Laurenzano et al., "PMaC's green queue: A framework for selecting energy optimal DVFS configurations in large scale MPI applications," Submission to CCPE Special Issue on Analysis of Performance and Power for Highly Parallel Systems, 2012.

[35] Y. Dong, J. Chen, X. Yang et al., "Energy-oriented openmp parallel loop scheduling," in Parallel and Distributed Processing with Applications, 2008. ISPA'08. International Symposium on. IEEE, 2008.

[36] P. Gschwandtner, J. J. Durillo, and T. Fahringer, Multi-Objective Auto-Tuning with Insieme: Optimization and Trade-Off Analysis for Time, Energy and Resource Usage. Cham: Springer International Publishing, 2014, pp. 87-98. [Online]. Available: http://dx.doi.org/10. 1007/978-3-319-09873-9_8

[37] K. Hoste and L. Eeckhout, "Cole: compiler optimization level exploration," in Proc. of the 6th Intl. Symposium on Code generation and optimization. ACM, 2008.

[38] L. Luo, Y. Chen, C. Wu, S. Long, and G. Fursin, "Finding representative sets of optimizations for adaptive multiversioning applications," arXiv preprint arXiv:1407.4075, 2014.

[39] G. Fursin, A. Memon, C. Gillon, and A. Lokhmotov, "Collective mind, part ii: Towards performance- and cost-aware software engineering as a natural science," in 18th International Workshop on Compilers for Parallel Computing (CPC15), 2015.

[40] V. Freeh and D. Lowenthal, "Using multiple energy gears in mpi programs on a power-scalable cluster," in Proc. of the 10th ACM SIGPLAN PPoPP. ACM, 2005.

[41] P. Balaprakash, A. Tiwari, and S. Wild, "Multi-objective optimization of hpc kernels for performance, power, and energy," in 4th International Workshop on Performance Modeling, Benchmarking, and Simulation of HPC Systems (PMBS12), 2013.

[42] P. A. Kulkarni, D. B. Whalley, G. S. Tyson, and J. W. Davidson, "Practical exhaustive optimization phase order exploration and evaluation," ACM Trans. Archit. Code Optim., vol. 6, no. 1, pp. 1:1-1:36, Apr. 2009. [Online]. Available: http://doi.acm.org/10.1145/ 1509864.1509865

[43] P. de Oliveira Castro, Y. Kashnikov, C. Akel, M. Popov, and W. Jalby, "Fine-grained benchmark subsetting for system selection," in Proceedings of Annual IEEE/ACM International Symposium on Code Generation and Optimization, ser. CGO '14. New York, NY, USA: ACM, 2014, pp. 132:132-132:142. [Online]. Available: http://doi.acm.org/10.1145/2544137.2544144 